\title{
Iterative PEEC-based Power Electronic Systems Simulations Using Reluctance and Regularization Techniques
}

\author{
Danesh Daroui \\ Department of Computer Science, \\ Electrical and Space Engineering \\ Luleå University of Technology \\ 97187 Luleå, Sweden \\ Email: danesh.daroui@ltu.se
}

\author{
Jonas Ekman \\ Department of Computer Science, \\ Electrical and Space Engineering \\ Luleå University of Technology \\ 97187 Luleå, Sweden \\ Email: jonas.ekman@ltu.se
}

\begin{abstract}
This paper presents a method to deal with the illposed and rank-deficient linear systems arising from accelerated partial element equivalent circuit-based electromagnetic simulations via a reluctance method. Since conventional, direct methods, cannot be used to solve these kind of problems, regularization techniques need to be employed. Among various regularization techniques, a least square-based method entitled LSQR is utilized to solve the rank-deficient problems. The proposed method is specially proper for the models where capacitive couplings can be neglected, since magnetic field is the dominating factor, like problems within power electronics area. The correctness of the presented PEEC-based solver is ensured by studying bus bar models which are a part of a frequency converters with the application in power electronics.
\end{abstract}

\section{INTRODUCTION}

Trends in modern electronic industry is toward high frequency electronic devices. High frequencies can give unwanted magnetic and electric couplings within and between components in a circuit, system or device. Studying these effects can be done using measurements, analytical calculations, and/or numerical techniques. Among the numerical techniques, the partial element equivalent circuit (PEEC) method [1], [2] is well-known and suitable for combined electromagnetic and circuit analysis and has been widely used in power electronics, antenna design and other industrial applications.

This paper deals with accelerating a PEEC-based solver using a reluctance technique [3] which provides the possibility to safely sparsify the partial inductance matrix in PEEC modeling. The result of this type of sparsification is intended to be numerically stable and give a valid solution with a controllable level of approximation. This leads to a smaller problem in sense of memory consumption and a more efficient solution by applying appropriate technique. On the other hand, the sparsified system is usually severely ill-posed so that conventional solvers cannot be employed to get stable results [4]. Moreover, due to very large condition numbers of the ill-posed problems, the solution is very sensitive to small perturbations of the original problem [5]. This means that even round-off errors within computer calculations can lead to numerical instability. Thus, regularization techniques should be applied in order to find a solution which is as accurate as possible [4], [6], [7].

In this paper, first the PEEC method is described and then the sparsification by reluctance theory. The problems which arise in PEEC simulations are numerically analysed, to identify the type of ill-posed problems, and then the appropriate regularization approach is discussed. At the end, some numerical results are given in order to prove the correctness of the proposed approach.

\section{PEEC THEORY}

The PEEC formulation [1] uses an integral equation solution of Maxwell's equations, which is interpreted as an equivalent circuit. To translate an electromagnetic problem into the circuit domain has several advantages. For example, the same model can be used for both time and frequency domain analysis (compare the .TRAN and .AC simulation option in classical SPICE solvers) and additional lumped components can easily be included. Further, the concept of electric equivalent circuits can be easier to understand than electromagnetic theory.

The concept of PEEC is shown in Fig. 1 where a simple PEEC model (b) has been constructed for a metal strip (a). The model consists of self partial inductances and capacitances (mutuals not shown) and volume cell resistances. A simulation carried out by the PEEC method consists of the following basic steps: (1) geometry description, (2) meshing, (3) placing additional components (e.g. passive components, sources, terminations, and ground), (4) calculation of partial elements $\left(\mathrm{R}, \mathrm{L}_{p}, \mathrm{C}\right)$ and connectivity information, (5) setting up and solving the time or frequency domain circuit equations, and (6) post-processing if field variables have to be calculated from the (now) known current and potential distribution.

Further PEEC theory and the enhancement to nonorthogonal geometries are given in [2]. 


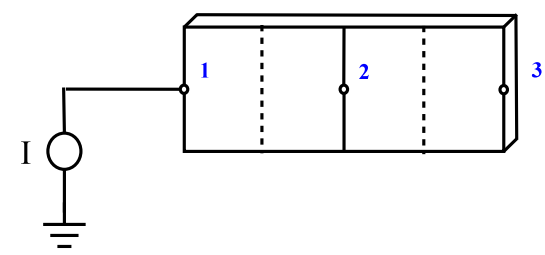

(a)

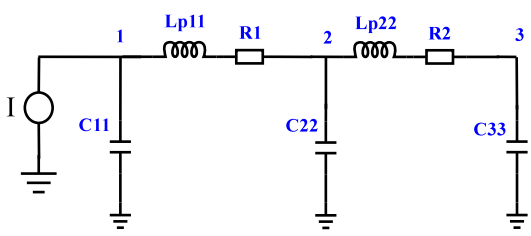

(b)

Fig. 1. Metal strip with 3 nodes and 2 cells (a) and corresponding PEEC circuit (b) (mutual couplings are not shown).

\section{RELUCTANCE FORMULATION AND REGULARIZATION}

In many applications, specially those which are involved in power electronic system simulations, due to the high currents in the devices, magnetic fields dominate over electric fields. Thus, the complete (quasi-static) $\left(\mathrm{R}, \mathrm{L}_{p}, \mathrm{C}\right) \mathrm{PEEC}$ model can safely be reduced to $\left(\mathrm{R}, \mathrm{L}_{p}\right)$ PEEC where the electric field couplings have been neglected. By using the reluctance method, the system of linear equations in the PEEC, MNA formulation will become sparse. As a consequence of the sparsification, the resulted sparse system can be severely illposed and hence over- or underdetermined. Obviously, such system cannot be solved using straightforward direct solvers e.g. LU decomposition. Hence, regularization techniques have to be applied to get an approximate solution.

\section{A. Sparsifying the partial inductance matrix via a reluctance technique}

By having a partial inductance matrix $L_{p}$, the reluctance matrix $K$ is defined as the inverse of the partial inductance matrix i.e. $K=L_{p}^{-1}$. The multiplication of $L_{p}$ with a vector of $N$ branch currents, results a vector containing the drops of the magnetic vector potential along each segment, shown in (1).

$$
\begin{aligned}
& {\left[\begin{array}{cccc}
L_{11} & L_{12} & \cdots & L_{1 N} \\
L_{21} & L_{22} & \cdots & L_{2 N} \\
\vdots & \vdots & \vdots & \vdots \\
L_{N 1} & L_{N 2} & \cdots & L_{N N}
\end{array}\right]\left[\begin{array}{c}
i_{1} \\
i_{2} \\
\vdots \\
i_{N}
\end{array}\right]=\left[\begin{array}{c}
\sum_{i=1}^{N}\left(\int A_{1 i} \mathrm{~d} \vec{l}_{1}\right) \\
\sum_{i=1}^{N}\left(\int A_{2 i} \mathrm{~d} \vec{l}_{2}\right) \\
\vdots \\
\sum_{i=1}^{N}\left(\int A_{N i} \mathrm{~d} \vec{l}_{N}\right)
\end{array}\right]} \\
& {\left[\begin{array}{cccc}
K_{11} & K_{12} & \cdots & K_{1 N} \\
K_{21} & K_{22} & \cdots & K_{2 N} \\
\vdots & \vdots & \vdots & \vdots \\
K_{N 1} & K_{N 2} & \cdots & K_{N N}
\end{array}\right]\left[\begin{array}{c}
\sum_{i=1}^{N}\left(\int A_{1 i} \mathrm{~d} \vec{l}_{1}\right) \\
\sum_{i=1}^{N}\left(\int A_{2 i} \mathrm{~d} \vec{l}_{2}\right) \\
\vdots \\
\sum_{i=1}^{N}\left(\int A_{N i} \mathrm{~d} \vec{l}_{N}\right)
\end{array}\right]\left[\begin{array}{c}
i_{1} \\
i_{2} \\
\vdots \\
i_{N}
\end{array}\right]}
\end{aligned}
$$

In order to extract the values of the reluctance matrix, the linear equation system (2) should be solved. Therefore, unlike for the partial inductances, there is no formulation to calculate reluctance values directly and the $L_{p}$ matrix is needed, to calculate the $K$ matrix. The physical meaning of $K_{i j}$ is defined by the induced current in the $i^{\text {th }}$ conductor (aggressor) when the total flux for the $j^{\text {th }}$ conductor (victim) is equal to one and those along all other conductors are set to zero. This definition yields, that the induced current in victims should be in opposite direction, comparing the current in the aggressor, to keep the flux around all victims to be zero. Furthermore, these induced currents will generate magnetic fields around victims which cancel the part of the field induced on the aggressor and shields the field on the aggressor to go further.

As for the capacitance matrix, the reluctance matrix is supposed to be symmetric, positive definite and having the locality property [3]. Hence, it is only needed to keep small number of elements in $K$ and still maintaining an appropriate level of accuracy. The stability of the method is directly related to how the structure is discritized. It has been proven that the stability is assured for a sufficiently discretized structure [8].

Based on the MNA formulation in PEEC, the system equation for a $\left(\mathrm{R}, \mathrm{L}_{p}\right)$ PEEC model is defined as

$$
\left[\begin{array}{cc}
Y_{c} & -A \\
A^{T} & R+j \omega L_{p}
\end{array}\right]\left[\begin{array}{l}
i \\
v
\end{array}\right]=\left[\begin{array}{c}
I \\
V
\end{array}\right]
$$

where $L_{p}$ is the partial inductance matrix, $R$ is the diagonal matrix of cell resistances, and $A$ is the connectivity matrix which describes the current direction between each pair of nodes assigned to each cell. All external lumped elements connected to the PEEC model are described in the admittance matrix $Y_{c}$. In the system equation (3), the right-hand-side is for excitation sources and the unknowns in the equation are node potential and cell currents.

Because of the locality properties of the reluctance matrix, it can safely sparsified by a truncation process, while still keeping the solution valid and accurate [9]. Later, by applying the sparsified reluctance matrix to both sides of (3), the new formulation which is shown in (4), will be achieved. This new formulation is introduced as $(\mathrm{R}, \mathrm{K}) \mathrm{PEEC}$.

$$
\left[\begin{array}{cc}
Y_{c} & -A \\
K A^{T} & K R+j \omega I
\end{array}\right]\left[\begin{array}{l}
i \\
v
\end{array}\right]=\left[\begin{array}{c}
I \\
K V
\end{array}\right]
$$

Obviously the approximation in (4) comes from the relation in (5) where the reluctance matrix is no longer the exact inverse of the partial inductance matrix. The new system of linear equations (4) is ill-posed and will need to be regularized before it is solved.

$$
K L_{p} \approx I
$$

\section{B. Rank-deficient ill-posed problems}

Ill-posed problems, represent a class of problems, where the condition number of the coefficient matrix $A$ in a linear system of equations $A x=b$ is very large. This class of problems is divided into two main categories i.e. rank-deficient and discrete ill-posed problems [4]. For an ill-posed system, the very large condition number, gives a hint that there exist at 
least some equations that are numerically dependent. Therefore, using conventional methods e.g. Gaussian elimination can fail to result a correct and stable solution. Additionally, linear systems which are severely ill-conditioned, are very sensitive to any small changes in the coefficient matrix, and any perturbation e.g. rounding errors can cause drastic changes in the solution and lead to totally invalid results.

Numerical tools such as Singular Value Decomposition (SVD) can help to identify the linear dependencies and then modify the system to get a better conditioned system. SVD can also be useful to determine whether an ill-posed problem is rank-deficient or discrete ill-posed. This can be done by analysing the singular values spectrum of the coefficient matrix where the gaps between large and small singular values mean that the matrix $A$ is rank-deficient. Figure 2 depicts the singular values of a coefficient matrix arose from discretization of integral equations in PEEC. It can be observed that multiple gaps (low index region) create distinguishable clusters between singular values which means that the coefficient matrix is not full-rank. Furthermore, Fig. 3 reveals that the Picard condition which is necessary for a discrete ill-posed problem is not satisfied. According to the Picard condition, if matrix $A$ in $A x=b$ is decomposed to $A=U S V^{*}$ using SVD, Fourier coefficients which are the elements of $U^{T} b$ should decay faster than singular values $\sigma_{i}$ [4]. It is clear that in Fig. 3, singular values decrease substantially faster than Fourier coefficients which confirms that the ill-posed problem belongs to the rankdeficient class.

It is also known that linear systems from discretized integral equations can easily lead to an ill-posed system such as Fredholm integral equations of the first kind [10]. Moreover, the source which makes a problem ill-posed can be due to incorrect mathematical modelling or applying coarse mesh over the structure when PEEC method is used. However, analysing these reasons are out of scope of this study.

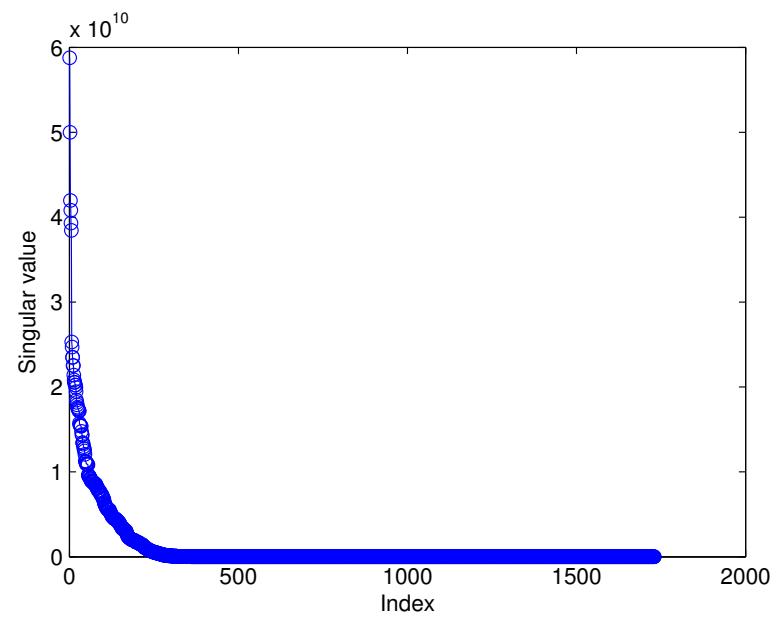

Fig. 2: Singular values of a rank-deficient problem in PEEC simulations.

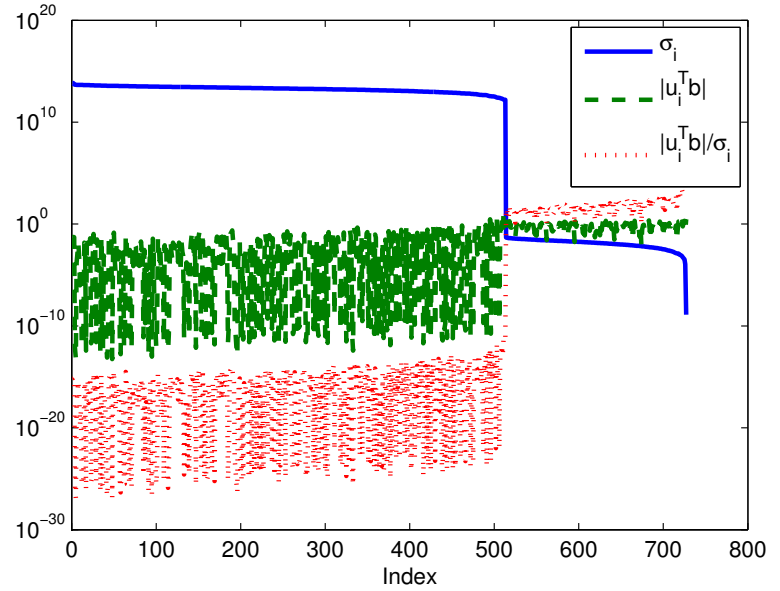

Fig. 3: Picard plot of a coefficient matrix in PEEC.

\section{Regularization techniques}

Several regularization techniques like Tikhonov [6], Truncated Singular Value Decomposition (TSVD), and Least Square methods [4] are available while in this paper, a method called LSQR is used. Tikhonov approach needs an optimal regularization parameter which can be determined using different numerical approaches, namely L-curve [11] which needs extensive analysis of the problem. TSVD also needs a low rank parameter i.e. $k$, where first largest $k$ singular values are kept and the rest will be set to zero. Regarding Fig. 2 the analysed system is so called an ill-determined system where by truncating the relatively smaller singular values, most of the singular values will be removed [12]. On the other hand, there is no well-defined gap in the largest group of the singular values which decade slowly. TSVD is appropriate for well-determined systems and therefore it can not be used for an ill-determined system where finding a proper value for $k$, to minimize the effect of perturbation which will happen by removing singular values is not possible. Moreover, it is proven that TSVD is not an appropriate method to be applied on the matrices arising from the discretization of integral equations [13]. Since PEEC is an integral equation-based technique, so TSVD can not be employed for regularization purposes when PEEC problems are being sovled. LSQR is instead attractive because it can be used for ill-determined rank-deficient systems. The original algorithm also includes the possibility for adding Tikhonov regularization with a fixed regularization parameter to the least square problem, but for simplicity this feature is not discussed in this paper.

LSQR is an iterative method, given for solving $A x=b$ and $\min \|A x-b\|_{2}$, where $A$ is large and sparse. The method is based on the bidiagonalization of Golub and Kahan [14] and is analytically equivalent to the conjugate gradient (CG) method. LSQR is appropriate for rank-deficient systems, however this condition is not essential. The algorithm is similar in style to CG method as applied to least squares problem. The matrix $A$ is used only to compute products of the form $A v$ and $A^{T} u$ 
for various vectors $v$ and $u$. Hence, $A$ will normally be large and sparse or will be expressible as product of matrices that are sparse or have special structure [7].

In this method a sequence of approximations $\left\{x_{k}\right\}$ is generated such that the residual norm $\left\|r_{k}\right\|_{2}$ decreases monotonically, where $r_{k}=b-A x_{k}$. Analytically, the sequence $\left\{x_{k}\right\}$ is identical to the sequence generated by the standard CG method, while LSQR is shown by example to be numerically more reliable in various circumstances than other CGlike methods [7]. The computational complexity of LSQR is expressed as $O(K N)$ where $K$ is number of iterations which cannot be larger than leading dimension of the coefficient matrix and $N$ is the number of non-zeros in the coefficient matrix [15]. Consequently, this method is appropriate for large and sparse systems where the complexity of the solution can be held still lower than direct factorization methods, due to the small number of non-zeros in the coefficient matrix.

\section{NUMERICAL RESUlts}

In order to study the accuracy and efficiency of the proposed method in this paper, a DC-link bus bar of a medium voltage frequency converters has been modelled as the case study [16]. The purpose of the DC-link in a frequency converter is to store the energy between the front-end and back-end inverter units. The selected frequency converter is rated for medium voltage applications with a nominal output voltage of $3.3 \mathrm{kV}$ and a nominal power of up to $32 \mathrm{MVA}$. For this power range several inverter units and low resistive film-capacitors are connected in parallel to the same DC-link. As a result of the inverter topology used, the DC-link must provide three voltage levels: the positive DC voltage (UDC+), the negative DC voltage (UDC-), and the neutral point (UDCn). Accordingly, the bus bar design consists of three parallel aluminium bars of $5.5 \mathrm{~m}$ length, connecting the capacitors and inverters throughout the entire converter cabinet. The design requirements to the bus bar are low resistivity for minimal losses and low inductivity to avoid unwanted resonances with the paralleled capacitors. Figure 4 depicts the modelled bus bars in PEEC. The capacitor connections are located at the two ends of the bars.

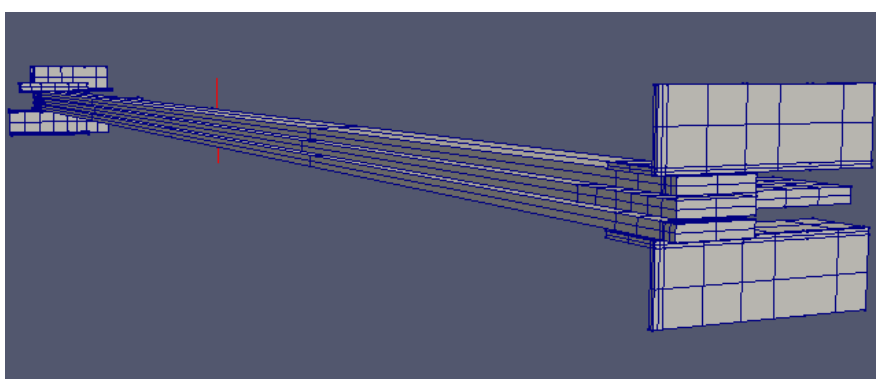

Fig. 4: Bus bar model in PEEC.

The simulated model consists of three parallel bars where upper- and lowermost bars are connected through a current source and a resistor at the near and far-end, respectively. The simulation has been performed to study the skin and proximity effects in the bars and simulate input resistance and inductance of the connected bus bars in a range of frequency between 1 and $100 \mathrm{KHz}$. The simulation results presented in this paper, has been compared and verified with measurements which is studied in [17].

The regularization routines have been invoked from the MATLAB package, Regularization Tools [18], which is a collection of MATLAB functions for analysis and solution of ill-posed problems. The regularization results when the coefficient matrix is not sparsified (i.e. 0\% sparse) have been compared with the results from a PEEC-based solver implemented in $\mathrm{C}++$, which utilizes direct solver with $\mathrm{LU}$ factorization. It was demonstrated that for $0 \%$ sparse systems and using LSQR method, the solution completely matched with the solution from implemented solver. Since the routines are all implemented in MATLAB, the speed-up of the routines are not discussed in this paper, while memory consumption and validity of the computed results are studied. It is however expected to get a substantial improvement in solution time when LSQR method is implemented as a part of the solver, comparing to the available solver. All tests have been carried out on a system with two Intel $2.4 \mathrm{GHz}$ processor and with 4 GB memory installed.

Table I lists number of unknowns of each test case. The test cases are quite small due to memory limitations, since all simulation were run in MATLAB.

TABLE I: Characteristics of the simulated bus bar models.

\begin{tabular}{c||r|r|r}
\multicolumn{1}{c||}{ Model } & \multicolumn{3}{c}{ Number of } \\
& $\begin{array}{r}\text { volume cells } \\
\left(N_{i}\right)\end{array}$ & $\begin{array}{r}\text { nodes } \\
\left(N_{\phi}\right)\end{array}$ & $\begin{array}{r}\text { total unknowns } \\
\left(N_{i}+N_{\phi}\right)\end{array}$ \\
\hline \hline BB1 & 1344 & 386 & 1730 \\
BB2 & 3412 & 908 & 4320
\end{tabular}

Table II also lists the memory consumption for each model. It is evident that from $0 \%$ to $95 \%$ sparsification, the memory has been reduced by factor of $\approx 13$. Continuing further sparsification from $95 \%$ to $98 \%$, the memory usage will reduce to $\frac{1}{3}$.

TABLE II: Memory usage of each simulated model.

\begin{tabular}{c||r|r} 
Model & Sparsified [\%] & Memory [MB] \\
\hline \hline BB1 & 0 & 77.5 \\
BB1 & 95 & 6.1 \\
BB1 & 98 & 2.3 \\
BB2 & 0 & 485.5 \\
BB2 & 95 & 37.5 \\
BB2 & 98 & 13
\end{tabular}

Figures 5 and 6 depict simulated resistance and inductance for $\mathrm{BB} 1$ and $\mathrm{BB} 2$, respectively. It is evident that skin and proximity effects have correctly been simulated for the tested bus bar models. According to the demonstrated results, the solution of $98 \%$ sparsified model from LSQR solver, agrees very well with the exact solution (i.e. $0 \%$ sparse) for all simulations. Despite small errors in resistance for BB2, the results are still accurate and quite stable.

Regarding the simulation results, it can be concluded that PEEC with reluctance technique will need only small part of 


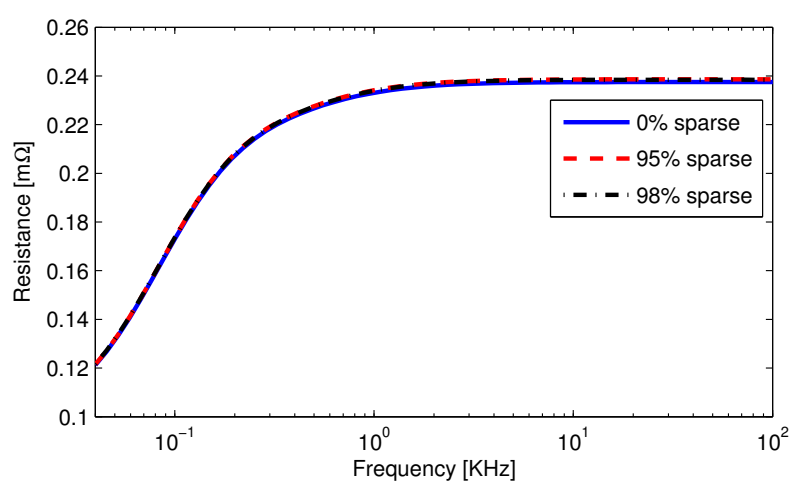

(a)

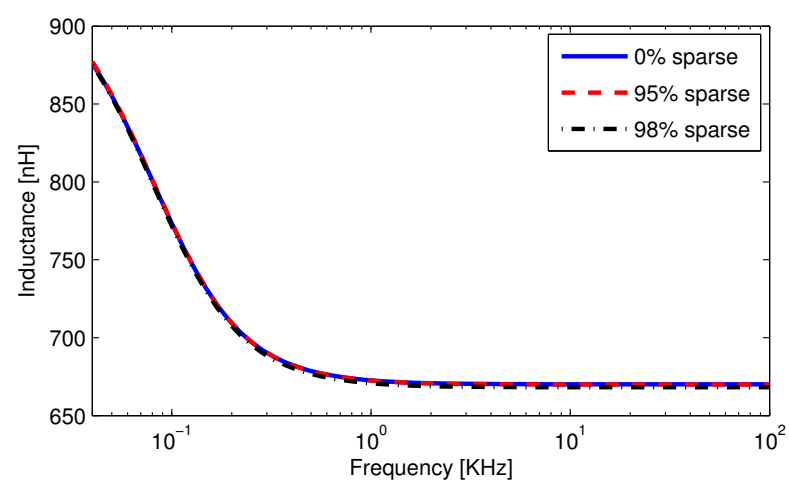

(b)

Fig. 5. Simulated bus bar resistance (a) and inductance (b) for BB1 test case.

the data to give a valid solution to a problem. This is of course for the sake of the locality property of the reluctance matrix, explained in Sec. III-A which will reduce the memory requirements dramatically while the accurate solution is guaranteed.

\section{CONCLUSiON}

A PEEC-based solver together with the reluctance method has been developed. Reluctance method makes it possible to sparsify the coefficient matrix $A$ in linear system $A x=b$ in PEEC, while the accuracy of the solution is guaranteed. The new sparse system is ill-posed and rank-deficient. Rankdeficient problems cannot be solved using typical direct solvers with LU factorization, due to over- or underdetermined system. Accordingly, regularization techniques need to be applied to get a valid solution for the sparse and ill-posed problems. The rank-deficient problem is solved using a least square-based method, namely LSQR which is an iterative approach and analytically equivalent to the conjugate gradient (CG) method . The validity of the method is confirmed by analyzing bus bar models which are used in power electronic medium voltage frequency converters. The newly introduced sparse solver opens the door to solve very large problems on computers with limited resources by reducing memory requirement.

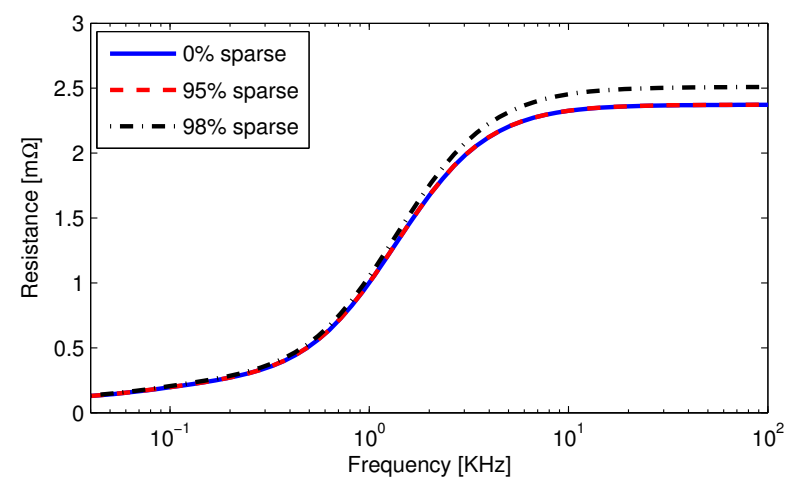

(a)

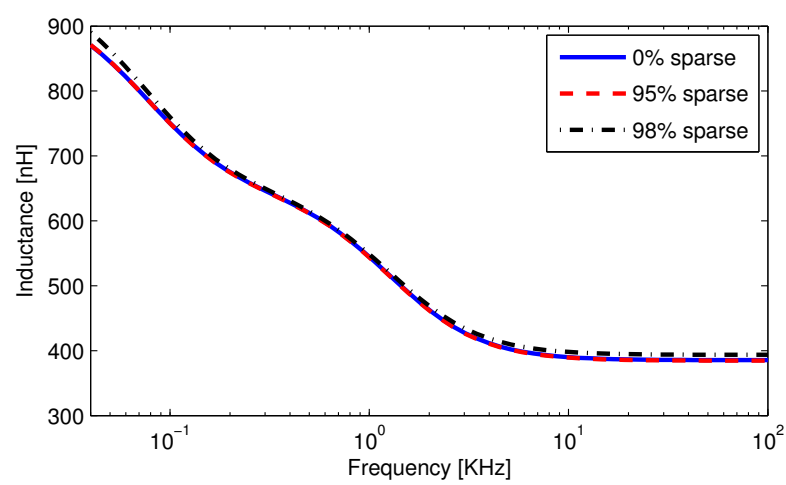

(b)

Fig. 6. Simulated bus bar resistance (a) and inductance (b) for BB2 test case.

\section{ACKNOWLEDGMENT}

The authors would like to thank ABB for providing bus bar models with measurements.

\section{REFERENCES}

[1] A. E. Ruehli, "Equivalent circuit models for three dimensional multiconductor systems," IEEE Trans. Microw. Theory Tech., Vol. 22, No. 3, pp. 216-221, 1974.

[2] A. E. Ruehli et al., "Non-orthogonal PEEC formulation for time and frequency domain EM and circuit modeling," IEEE Trans. on Electromagn. Compat., Vol. 45, No. 2, pp. 167-176, 2003.

[3] T. H. Chen, C. Luk, H. Kim, and C. P. Chen, "Inductwise: Inductancewise interconnect simulator and extractor," In Proc. of the IEEE Int. Conf. on Computer Aided Design, CA, USA, 2002.

[4] P. C. Hansen, "Rank-deficient and discrete ill-posed problems," SIAM, Philadelphia, 1998.

[5] C. D. Meyer, "Matrix analysis and applied linear algebra," SIAM, Philadelphia, 2000.

[6] A. N. Tikhonov and V. Y. Arsenin, "Solution of ill-posed problems," Winston, Washington, D.C., 1977.

[7] C. C. Paige and M. A. Saunders, "LSQR: an algorithm for sparse linear equations and sparse least squares," ACM Trans. Math. Software, Vol. 8, No. 1, pp. 43-71, 1982.

[8] H. Ji, A. Devgan, and W. Dai, "Ksim: A stable and efficient RKC simulator for capturing on-chip inductance effect," In Proc. of Design Automation Conference, CA, USA, 2002.

[9] M. L. Zitzmann, "Fast and efficient methods for circuit-based automotive EMC simulation," PhD thesis, University of Erlangen- Nürnberg, February 2007 
[10] H. Du and M. Cui, "Approximate solution of the Fredholm integral equation of the first kind in a reproducing kernel Hilbert space," Elsevier Applied Mathematics Letters, Vol. 21, No. 6, pp. 617-623, 2008.

[11] P. R. Johnston, "Selecting the corner in the L-curve approach to Tikhonov regularization," IEEE Trans. on Biomedical Engineering, Vol. 47, No. 9, pp. 1293-1296, 2000.

[12] P. C. Hansen, "The truncated SVD as a method for regularization," BIT Computer Science and Numerical Mathematics, Vol. 27, No. 4, 1987.

[13] B. W. Rust, "Truncating the singular value decomposition for ill-posed problems," National Institute of Standards and Technology (NIST), 1998.

[14] G. H. Golub and W. Kahan, "Calculating the singular values and pseudoinverse of a matrix," SIAM J. Numer. Anal., Vol. 2, pp. 205-224, 1965.

[15] T. Hrycak and G. Matz, "Low-complexity time-domain ICI equalization for OFDM communications over rapidly varying channels," In Proc. of The Fortieth Asilomar Conference on Signals, Systems \& Computers, CA, USA, 2006.

[16] Medium Voltage Power Converter Products. [Online]. Available: http://www.abb.com 2012.

[17] D. Daroui, I. Stevanović, D. Cottet, and J. Ekman, "Bus bar simulations using the PEEC method," In Int. Review of Progress in Applied Computational Electromagnetics ACES, Tampere, Finland, 2010.

[18] Regularization Tools HomePage. [Online]. Available: http://www2.imm.dtu.dk/ pch/Regutools/ 2012. 